\title{
Cocaine or Selective Block of Dopamine Transporters Influences Multisecond Oscillations in Firing Rate in the Globus Pallidus
}

\author{
David N. Ruskin, Ph.D., Debra A. Bergstrom, Ph.D., David Baek, B.S., Lauren E. Freeman, B.S., \\ and Judith R. Walters, Ph.D.
}

\begin{abstract}
Previous studies have shown that direct-acting dopamine agonists modulate the multisecond oscillations which are present in globus pallidus spike trains in vivo in awake rats. To investigate possible modulation by endogenous dopamine and by other monoamines, and by drugs with abuse potential, cocaine or selective monoamine uptake blockers were injected systemically during extracellular recording of single globus pallidus neurons and the results analyzed with spectral and wavelet methods. Both cocaine and the selective dopamine uptake blocker GBR-12909 significantly shortened the period of multisecond oscillations, as well as increasing overall firing rate. Cocaine effects were blocked by dopamine antagonist
\end{abstract}

pretreatment, as well as by $\mathrm{N}$-methyl-D-aspartate receptor antagonist (MK-801) pretreatment. Desipramine and fluoxetine (blockers of norepinephrine and serotonin uptake, respectively) had no significant effects on multisecond oscillations. The results suggest that dopamine has a primary role among monoamines in modulating multisecond oscillations in globus pallidus activity, and that tonic dopaminergic and glutamatergic transmission is necessary for normal slow oscillatory function.

[Neuropsychopharmacology 25:28-40, 2001] (C) 2001 American College of Neuropsychopharmacology. Published by Elsevier Science Inc.
KEY WORDS: Cocaine; Dopamine; GBR-12909; Globus pallidus; NMDA receptor; Oscillation; Wavelet

Periodicities in brain electrical activity occur in many nuclei and in many frequency ranges, and have been described in the electroencephalogram, in field potentials, and in the spiking activity of single neurons. Much of the attention that brain periodicities have received has been directed to the range of frequencies

From the Experimental Therapeutics Branch, National Institute of Neurological Disorders and Stroke, National Institutes of Health, Bethesda, MD.

Address correspondence to: David N. Ruskin, 10 Center Dr., Room 5C103, Bethesda, MD, 20892-1406, Tel.: 301-496-2067, Fax: 301-496-6609, E-mail: ruskind@ninds.nih.gov

Received Jun 22, 2000; revised September 22, 2000; accepted November 17, 2000. from $1 \mathrm{~Hz}$ (the slow end of the delta rhythm) up to roughly $60 \mathrm{~Hz}$ (the fast end of the gamma rhythm). However, recent studies have demonstrated that much slower periodic oscillations are commonly present in the spike trains of tonically firing neurons in the basal ganglia. The firing rates of a majority of neurons in the globus pallidus (GP), the subthalamic nucleus, the substantia nigra pars reticulata and the entopeduncular nucleus oscillate in vivo with slow periods, most often in the range of 10-60 s (0.1-0.017 Hz) (Ruskin et al. 1999a; Ruskin et al. 1999c; Wichmann et al. 1999; Allers et al. 2000). These multisecond periodicities in tonic activity are present in awake rats, but are markedly attenuated by general anesthesia (Ruskin et al. 1999a).

One of the most striking characteristics of these slow oscillations is their strong modulation by dopamine 
(DA) receptor activation (Ruskin et al. 1999a; Ruskin et al. 1999c; Allers et al. 2000). Systemically injected DA agonists shift oscillatory periods from mean periods of $25-30 \mathrm{~s}$ to $5-15 \mathrm{~s}$, depending on drug and dose. Spectral analysis reveals that DA receptor activation also increases the spectral power (i.e. the regularity) of multisecond periodicities. Therefore, in addition to the wellcharacterized ability of systemically-administered DA agonists to change the mean firing rate of basal ganglia neurons, they also change basal ganglia firing patterns at multisecond time scales. The use of selective DA receptor agonists and antagonists has shown that this modulation of slow firing pattern involves both the $\mathrm{D}_{1}$ and $\mathrm{D}_{2}$ subfamilies of DA receptors (Ruskin et al. 1999a; Ruskin et al. 1999c).

These initial studies of DAergic influences on multisecond periodicities utilized direct-acting DAergic drugs. More recently, we have found that D-amphetamine and methylphenidate, drugs that act on monoamine transmission indirectly, also modulate slow oscillations (Ruskin et al. 2001). These drugs are noted for their effects on attention and locomotor activity, including beneficial effects on these parameters in patients with attention deficit/hyperactivity disorder. D-amphetamine and methylphenidate also have serious abuse potential. To further explore the potential for abused/addictive substances to affect multisecond oscillatory activity, the present study examined the effects of cocaine on spiking activity in the GP. Since these three drugs raise levels of more than one brain monoamine, this study also tested the effects of the selective uptake blockers GBR-12909, desipramine and fluoxetine (selective for the DA, norepinephrine (NE) and serotonin (5-HT) uptake systems, respectively) to determine the relative importance of each monoamine. In addition, because many of the effects of direct-acting dopamine agonists on mean firing rate in the basal ganglia depend on $N$-methyl-D-aspartate (NMDA) receptor activity (Kelland and Walters 1992; Allers et al. 1996; Huang et al. 1998), the NMDA receptor antagonist MK801 was utilized to test for a similar dependence of the effects of monoamine uptake blockade on multisecond periodicities.

\section{METHODS}

Extracellular single unit activity was recorded in the GP of male Sprague-Dawley rats (250-400 g) in accordance with the National Institutes of Health (USA) Guide for the Care and Use of Laboratory Animals. In previous studies of GP unit activity, we have found that the incidence of multisecond oscillations was greatly attenuated when animals were under general anesthesia (Ruskin et al. 1999a). Therefore, in the present study GP neurons were recorded in awake, immobilized rats as previously described (Ruskin et al. 1999a). Briefly, all surgical procedures (tracheotomy, scalp incision, skull drilling) were performed with rats under halothane anesthesia. Halothane anesthesia was induced in an induction chamber, and was maintained during surgery by administration of additional halothane as needed. To prevent discomfort, all surgical sites and pressure points were injected with the long-acting local anesthetic mepivacaine, and ear bar tips and tracheal cannulas were coated with $2 \%$ lidocaine anesthetic gel. Corneal drying was prevented with LacriLube. A tracheotomy was performed, and the trachea was cannulated. Rats were placed in a stereotaxic frame, and a hole was drilled in the skull over the GP. After surgical procedures were finished, halothane anesthesia was discontinued, the animal was paralyzed with gallamine triethiodide $(16 \mathrm{mg} / \mathrm{kg}$ i.v.) injected through the tail vein, and artificial respiration immediately begun with a rodent ventilator (model 683, Harvard Apparatus). Artificial respiration rates were adjusted to maintain exhaled $\mathrm{CO}_{2}$ between 3.6 and $4.5 \%$. Supplements of gallamine were given every 10-15 min. Body temperature was maintained at $36-38^{\circ} \mathrm{C}$ with a heating pad.

Glass microelectrodes $(2.5-6 \mathrm{M} \Omega$ at $135 \mathrm{~Hz})$ filled with $2 \mathrm{M} \mathrm{NaCl}$ containing 1\% Pontamine Sky Blue were lowered stereotaxically through skull holes to the following coordinates: $0.8-1.2 \mathrm{~mm}$ posterior to Bregma, 2.6-3.0 $\mathrm{mm}$ lateral to the midline, $5.0-7.0 \mathrm{~mm}$ ventral to dura. Fine vertical control of microelectrode placement was accomplished with micromanipulators (MO-8, Narashige). Single unit activity was amplified (model 725, WPI) and monitored with an audio monitor and digital oscilloscope. Signals were band-pass filtered between 250 and $5000 \mathrm{~Hz}$, and spikes were identified with a voltage/window discriminator and recorded by Spike2 software (version 3.14, Cambridge Electronic Design). All recorded GP units had biphasic +/waveforms (type II; Kelland et al. 1995), and had baseline firing rates between 13 and $92 \mathrm{~Hz}($ mean $=36 \mathrm{~Hz}$ ). Five to six minutes of baseline activity was recorded before the i.v. injection of experimental drugs through the tail vein. Drugs were injected as a single bolus, except for GBR-12909, which in some instances was injected in four divided doses at 1-min intervals. Units were further recorded for 10-20 min. In some cases, a second drug was then injected and units were held for another $10 \mathrm{~min}$. One unit was recorded per rat. After data collection, Pontamine Sky Blue was iontophoresed to mark the recording site for later histological confirmation.

Experimental drugs used include (-)-cocaine $\mathrm{HCl}$, desipramine $\mathrm{HCl}$ (Norpramin), S(-)-eticlopride $\mathrm{HCl}$, fluoxetine $\mathrm{HCl}$ (Prozac), GBR-12909 2:HCl (Vanoxerine), haloperidol, (+)-MK-801 hydrogen maleate (Dizocilpine), and $\mathrm{R}(+)-\mathrm{SCH}-23390 \mathrm{HCl}$. All experimental drugs were from Research Biochemicals Inc., except for haloperidol (McNeil Pharmaceuticals). Drugs were dissolved in 
$0.9 \% \mathrm{NaCl}$, except for fluoxetine and GBR-12909 which were dissolved in distilled water, and SCH-23390 which was dissolved as a 10X stock solution in $0.001 \mathrm{~N} \mathrm{HCl}$ before dilution to final volume with saline.

Spectral analysis of GP spike trains was performed by two methods. For assessing the significance of periodic activity, $180 \mathrm{~s}$ data segments from pre- and postdrug epochs were analyzed with the Lomb periodogram (Kaneoke and Vitek 1996), performed on binned spike trains. This method provides a measure of statistical significance for spectral features: spectra were tested against the null hypothesis that binned spiking activity had a random Gaussian distribution, resulting in an exponential distribution of spectral power. This null hypothesis was rejected at the $p=.01$ level. For spectral analysis, spiking activity was binned with $200 \mathrm{~ms}$ bins (example spike trains in figures are shown binned at $500 \mathrm{~ms}$ ), and periodicities were analyzed in the range of $1.5-60 \mathrm{~s}(0.67-0.017 \mathrm{~Hz})$. Periodicities slightly faster than $1.5 \mathrm{~s}$ in this preparation often relate to ventilation (Ruskin et al. 1999a). For example, of the presently recorded GP neurons, $11.4 \%$ had significant oscillations in this range, all having periods between 0.77 and $0.84 \mathrm{~s}$. This coincides with the range of ventilator speeds, and when ventilator action was recorded simultaneously during the recording of a neuron with this type of activity $(\mathrm{n}=5)$, the neuronal oscillatory period and the ventilator period were equal to within $1 \%$. These ventilator-related oscillations were not further analyzed. $180 \mathrm{~s}$ data segments were chosen from baseline, and from the 5-10 min and (when available) 15-20 min time ranges after drug injection. To assess drug effects on oscillatory period, the period of the "main" spectral peak, i.e. the tallest (most powerful) spectral peak within the studied range, was taken from the power spectrum of each analyzed segment, and these numbers were analyzed with ANOVA followed by post hoc Dunnett's comparisons to baseline. Data segments that had no significant periodicity in the 1.5-60 s range were not entered into this analysis. To assess drug effects on oscillatory regularity (i.e. spectral power) apart from effects on oscillatory period, the total area under the spectral curve within the 1.5-60 s range was measured, including those cases where there were no significant spectral peaks, and these data were analyzed with repeated-measures ANOVA followed by post hoc Dunnett's comparisons to baseline. The $\alpha$ level for ANOVA and post hoc tests was $p=.05$.

In a second method of spectral analysis, focusing on the evolution of periodic activity over time, entire recordings or recording segments were represented as time-frequency plots of spectral power. These scalograms were based on the continuous wavelet transform and are analogous to Fourier transform-based spectrograms. A wavelet-based approach was used to optimize time resolution across all examined frequencies, com- pared to the single time resolution of windowed Fourier transform analysis. Scalograms were produced with the Time-Frequency Toolbox (http://www-syntim. inria.fr/fractales/Software/TFTB) with Matlab 5.3.1 (The MathWorks), and were computed with the Morlet wavelet (15 s half-width at coarsest scale), with 256 frequencies being sampled within the analyzed range. Spiking activity was binned at $0.75 \mathrm{~s}$, to reach a Nyquist limit of $1.5 \mathrm{~s}(0.67 \mathrm{~Hz})$. Spectral power was plotted on a $\log _{10}$ scale, with greater power represented by redder color. The color axis was scaled so that the power levels within the scalogram data for a single neuron spanned the full color range (blue to red).

\section{RESULTS}

\section{Multisecond Periodicities in Baseline Activity}

As reported previously (Ruskin et al. 1999a; Ruskin et al. 1999c), the baseline firing rate of most GP neurons recorded in awake rats oscillated at time scales of seconds to minutes (Figures 1 and 2). These periodic variations could be large in amplitude: in the baseline segment shown in Figure 2, Panel A, the firing rate varies up to $180 \%$ and down to $60 \%$ of the overall mean. Using the Lomb algorithm to assess the periodicity of these oscillations (examples in Figure 2), 80\% of neurons displayed oscillations in baseline firing rate (with periods between 1.5 and $60 \mathrm{~s}$ ) that were sufficiently powerful (i.e., regular) to be considered statistically significant. Oscillatory periods of the most powerful spectral peak from each baseline power spectrum are plotted in Figure 3. These baseline oscillatory periods varied widely from neuron to neuron (within the 1.5-60 s range), but mean values for all treatment groups were approximately $30 \mathrm{~s}$ (28.2 \pm $2.1 \mathrm{~s}$ for all neurons combined).

\section{Effects of Selective Monoamine Uptake Blockers}

Most recorded units had significant slow oscillations both before and after the injection of selective monoamine uptake blockers (Table 1). However, among GBR12909, desipramine and fluoxetine, only the selective DA uptake blocker GBR-12909 robustly effected these oscillations. Particularly striking oscillations appeared after the i.v. injection of this drug $(3.2 \mathrm{mg} / \mathrm{kg})$, and, once they had appeared, could be remarkably stable (Figure 1). Spectral analyses indicated that these oscillations occurred at a faster rate compared to baseline (Figures 1 and 3, Panel A). The mean oscillatory period within the 5-10 min post-drug epoch was $\sim 10 \mathrm{~s}$, and this reduction in period was still significant out to 20 min after drug injection, at which time a DA antagonist was typically administered (Figures 1 and 3, Panel A). In addition, GBR-12909 increased the power (regularity) of the multisecond oscillations. This was visible in 


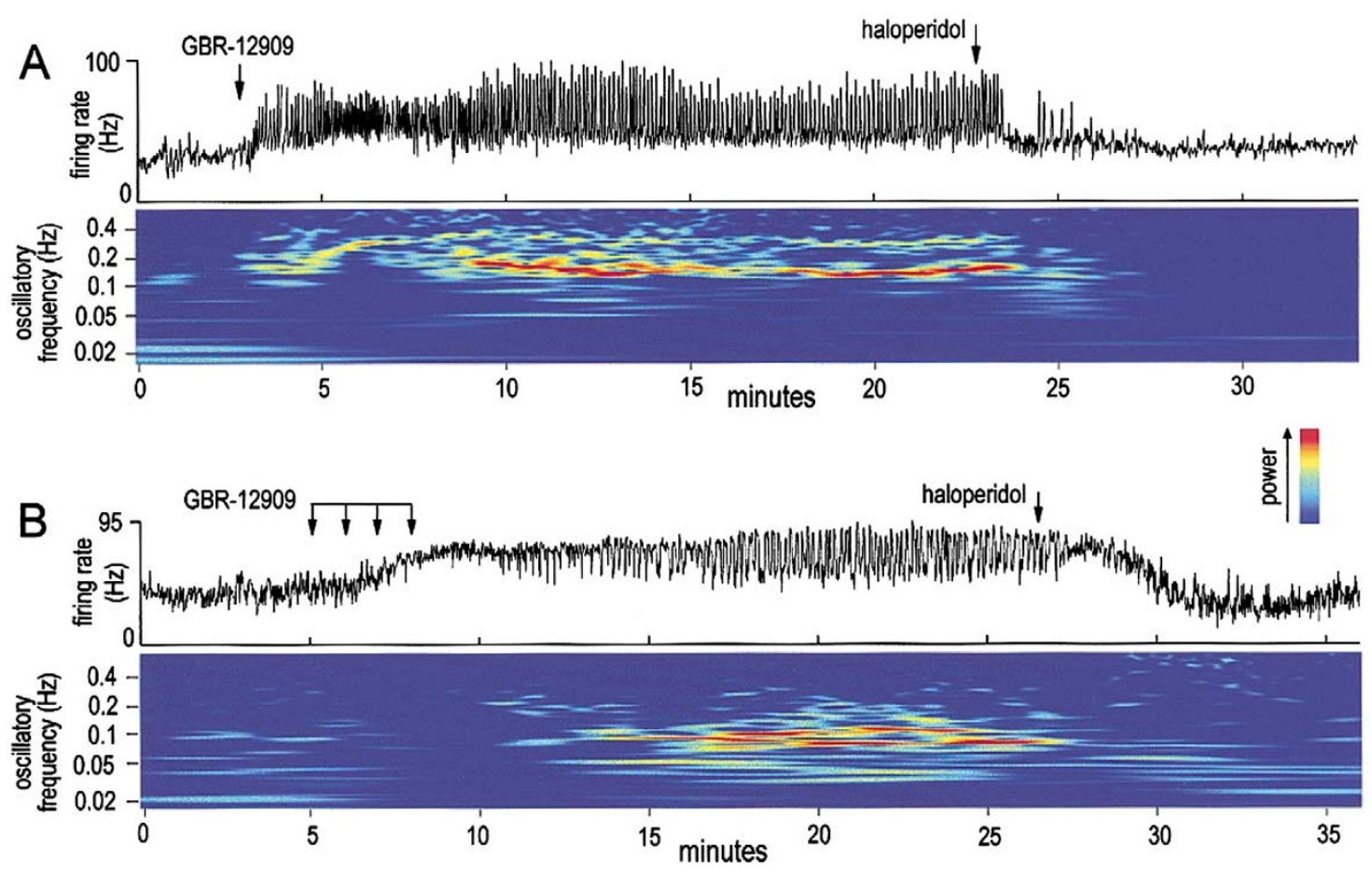

Figure 1. Effects of DA uptake blockade on multisecond oscillations in firing rate in GP neurons. Two complete firing rate records are shown, with accompanying wavelet-based time vs. frequency plots (scalograms) for illustration of oscillatory frequency between 0.67 and $0.017 \mathrm{~Hz}(1.5-60 \mathrm{~s})$. The frequency (y) axis of the scalograms is $\log _{10}(\mathrm{~Hz})$. Redder colors represent higher power (scale bar). Both neurons had somewhat irregular slow activity in baseline, which appears in the scalograms as low-power bands between 0.025 and $0.017 \mathrm{~Hz}$ (40-60 s). In both units, $3.2 \mathrm{mg} / \mathrm{kg}$ GBR-12909 (given as bolus dose in Panel A, divided doses in Panel B), caused the emergence of faster, and more regular, oscillations in firing rate which are readily apparent in the firing rate records. The scalograms illustrate the emergence of these oscillations as the appearance of powerful bands centered around $0.15 \mathrm{~Hz}(7.5 \mathrm{~s})$ in Panel A, and $0.1 \mathrm{~Hz}(10 \mathrm{~s})$ in Panel B. These faster oscillations appeared promptly after GBR-12909 injection in A, but were somewhat delayed in Panel B. GBR-12909 also increased average firing rate in both neurons. In both units, oscillatory activity remains fairly stable until the injection of $0.2 \mathrm{mg} / \mathrm{kg}$ haloperidol, a $\mathrm{D}_{2}$ antagonist, which reversed the effects of GBR-12909 on multisecond pattern and average firing rate.

scalograms as the emergence of higher power bands (Figure 1), and was quantified by the measurement of spectral area in Lomb algorithm-generated power spectra. GBR-12909 increased mean spectral power by almost $70 \%$ (Figure 4 ).

In contrast to GBR-12909, neither the NE uptake blocker desipramine nor the 5-HT uptake blocker fluoxetine had significant effects on oscillatory period (Figure 3, Panels B and C). Also, neither drug significantly affected spectral power (data not shown). After desipramine injection, there did appear to be a modest preponderance of oscillation periods below $\sim 20$ s. To address the possibility that this pattern might indicate the existence of a "desipramine-responsive" subpopulation, more detailed analyses were performed. Neurons were characterized as having high or low baseline firing rate (defined by a median split), or as responding to desipramine with increases or decreases in firing rate (compared to baseline). Separate ANOVAs on desipramine effects on oscillatory period for each of these groups were all non-significant. In addition, post-desipramine oscillatory period did not significantly correlate with baseline firing rate or with post-desipramine firing rate (as absolute rate or as percent of baseline). However, these data do not rule out the possibility of a "desipramine-responsive" subpopulation of neurons or rats.

In addition to effects on multisecond oscillations, GBR-12909 was the only selective monoamine uptake blocker to have robust effects on overall firing rate. This drug increased overall firing rates in all tested neurons, whereas desipramine and fluoxetine had only minor effects (Figure 5).

GBR-12909 (3.2 mg/ $\mathrm{kg})$ given i.v. to freely-moving rats induced a pattern of strong downward sniffing and/or chewing, sometimes combined with slow or intermittent locomotion. This pattern was present in both the 5-10 and 15-20 min post-drug time ranges.

\section{Effects of Cocaine}

Striking multisecond oscillations appeared after injection of the non-specific monoamine uptake blocker co- 

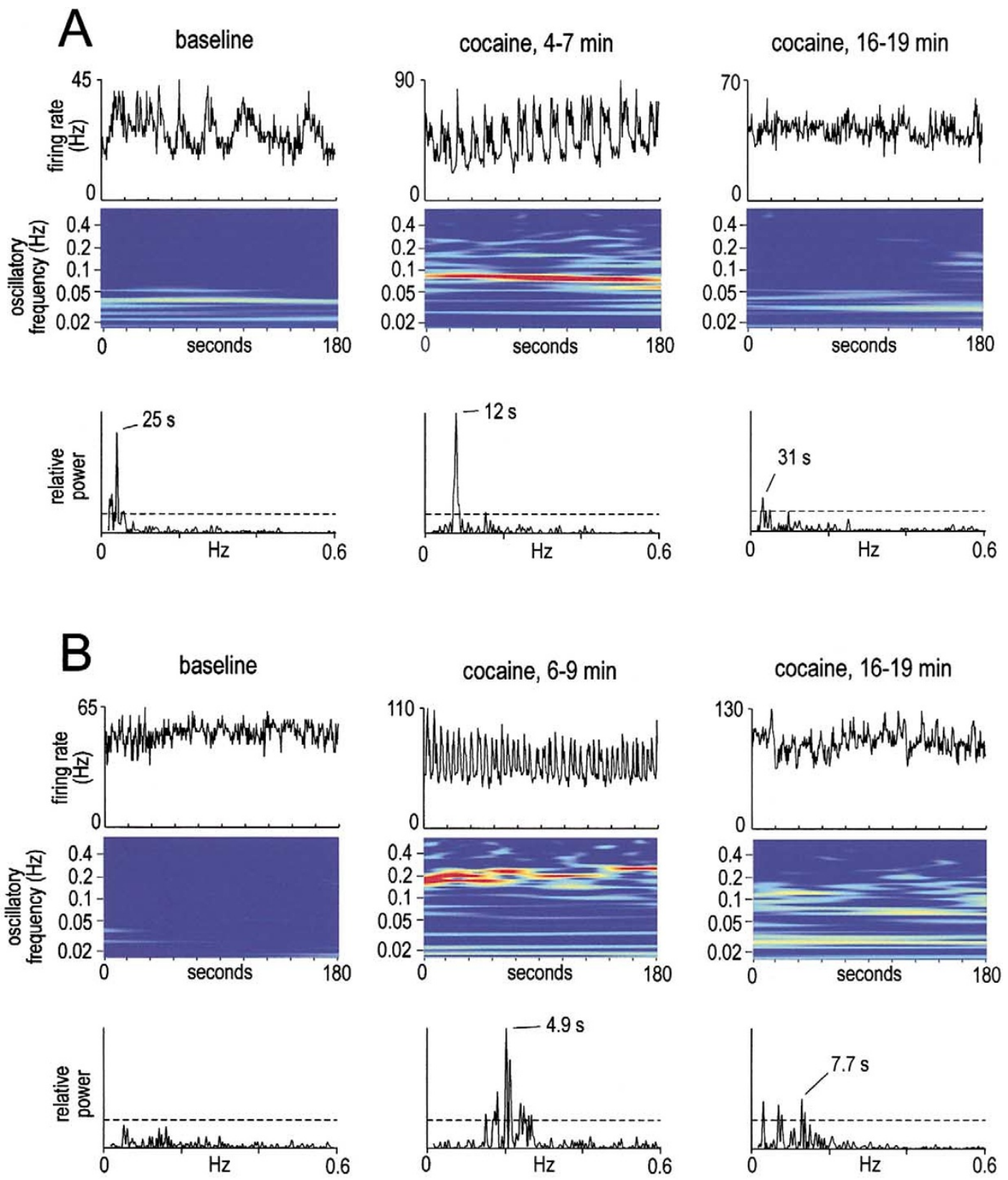

Figure 2. Effect of cocaine on oscillatory frequency in GP neurons. $180 \mathrm{~s}$ segments of binned spiking activity (scaled to their highest point) are shown from baseline and post-cocaine $(3.2 \mathrm{mg} / \mathrm{kg})$ epochs, with accompanying spectral analyses. Directly below the data segments (and plotted on the same time scale) are corresponding sections of the wavelet-based scalograms for each unit, showing oscillatory frequency and power over time. Conventions for scalograms are as in Figure 1; the color scale is identical for scalograms taken from a given unit. Below scalograms are power spectra derived from Lomb algorithm analyses on the same $180 \mathrm{~s}$ segments. These power spectra are shown with lines signifying the $p=.01$ significance level; spectral peaks above this line are considered statistically significant (see Methods). The period of the main (most powerful) significant spectral peak in each spectrum is indicated. Panel A: This unit had slow, somewhat irregular (but high amplitude) firing rate variations in baseline, visible in the scalogram and identified as having a main period of $25 \mathrm{~s}$ in the power spectrum. At 4-7 min after the injection of cocaine, oscillation period decreased to $12 \mathrm{~s}$, and power (regularity) increased. A very stable and powerful band is apparent in the scalogram. Later (16-19 min after injection), the effects of cocaine spontaneously reversed: slow oscillatory activity decreased in power, and increased in period. Panel B: This unit did not have significant oscillatory activity in baseline. At 6-9 min after cocaine injection, very regular oscillations were noted, identified in the power spectrum as having a main period of $4.9 \mathrm{~s}$. The accompanying scalogram indicates that the oscillation period varied somewhat over time. The effects of cocaine eventually wore off: 16-19 min post-drug, oscillatory power decreased (visible in both the scalogram and the power spectrum), and oscillatory activity became disorganized. Several peaks (and bands) are visible across a wide range of periods in the power spectrum (and the scalogram). Cocaine also increased average firing rates in both neurons. 

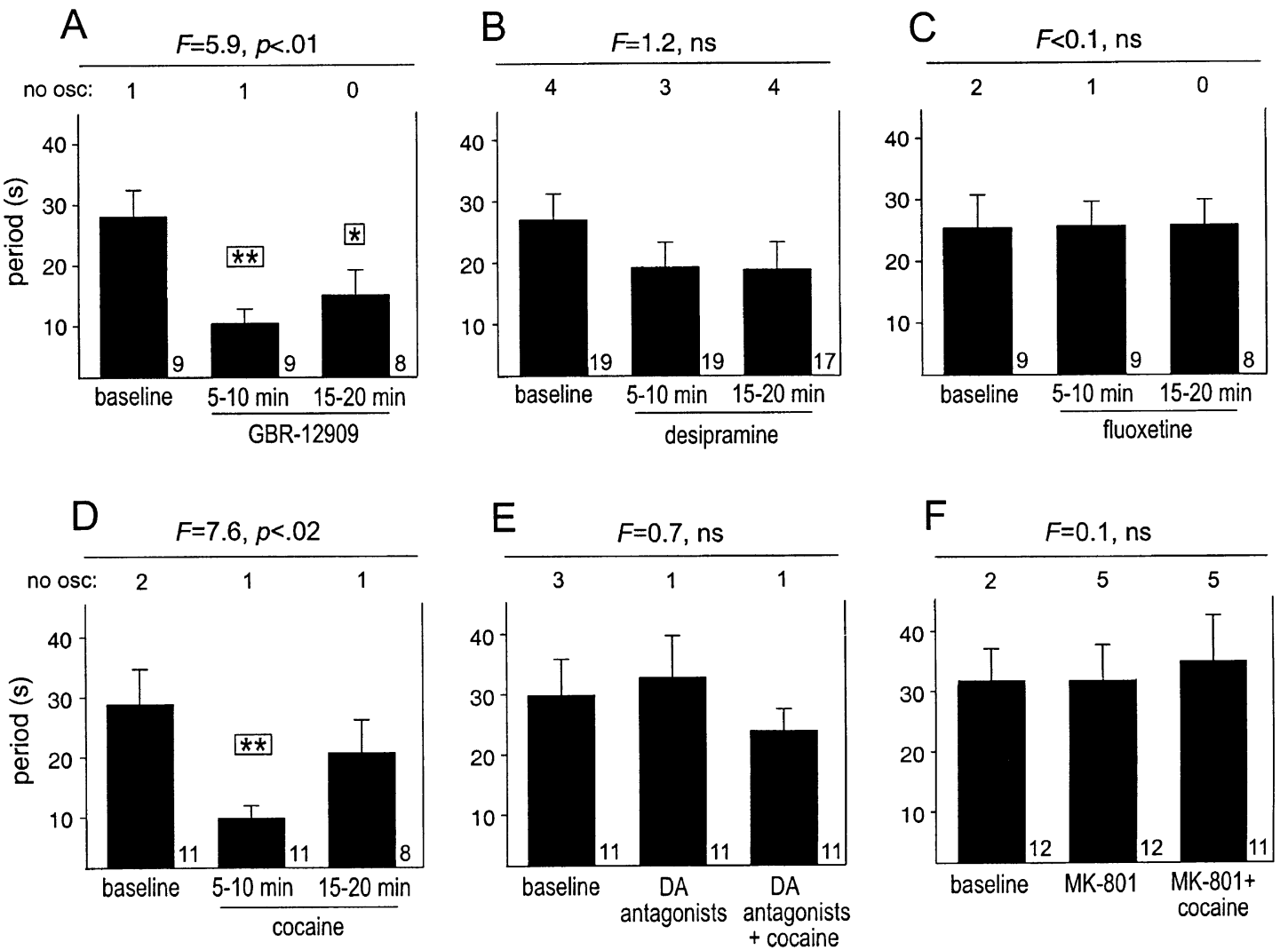

Figure 3. The effect of monoamine uptake blockers on oscillatory period, and the effect of pretreatment with DA receptor or NMDA receptor antagonists. Bars represent the mean \pm SEM of the periods of the most powerful Lomb spectral peaks (examples in Figure 2) of neurons within a particular condition. The total number of cells for each group is given at the base of each bar; the number of cells in the group that are without significant periodicity (i.e. no spectral peaks above the $p=.01$ significance level) is indicated above each bar ("no osc"). ANOVA results (F and $p$ values) for each treatment group are given above each graph. Panels A, B, C: Period was significantly reduced by GBR-12909 (3.2 mg/kg), but not by desipramine $(2.0$ and $5.0 \mathrm{mg} / \mathrm{kg})$ or by fluoxetine $(3.2 \mathrm{mg} / \mathrm{kg})$. Separate ANOVAs on desipramine at these two doses indicated no significant effect of either; therefore, the groups are combined here. Panel D: Period was also significantly reduced by cocaine (3.2 $\mathrm{mg} / \mathrm{kg}$ ), although this effect was no longer apparent in the 15-20 min time range. Panels E, F: The effect of cocaine was blocked by pretreatment with either combined $\mathrm{D}_{1}$ and $\mathrm{D}_{2}$ antagonists $(\mathrm{SCH}-23390,0.5 \mathrm{mg} / \mathrm{kg}$, and eticlopride, $0.2 \mathrm{mg} / \mathrm{kg}$; "DA antagonists") or by MK-801 $(0.15 \mathrm{mg} / \mathrm{kg})$. Cocaine data in Panels E and F are from the 5-10 min range. Neither the DA antagonist treatment or MK-801 had significant effects on period alone. ns: non-significant. ${ }^{*} p<.05,{ }^{* *} p<.02$.

caine (Figure 2), and like GBR-12909, cocaine reduced the periods of these oscillations to a mean of $\sim 10 \mathrm{~s}$ within the 5-10 min epoch (Figure 2 and 3, Panel D). However, cocaine effects on oscillatory period were short-lived, not lasting through the 15-20 min postdrug epoch, as illustrated by visual inspection of firing activity and scalograms (Figure 2), and confirmed by Lomb analysis (Figure 3, Panel D). Cocaine-induced increases in overall firing rate were also typically shortlived, reaching a plateau at 4-9 $\mathrm{min}$ and dropping thereafter. These results are consistent with the relatively short biological half-life of this drug in rats after i.v. administration (Barbieri et al. 1992). Unlike GBR12909, cocaine did not have significant effects on spectral power. Although some units responded to cocaine with an increase in power (e.g. Figure 2, Panel B: there is an $83 \%$ increase in spectral area (middle column) compared to baseline), there was no consistent power response (Figure 4).

Although the large majority of type II (+ /- waveform) GP neurons respond to indirect-acting or directacting DA agonists with net increases in firing rate, rare type II neurons are found that respond with decreased net firing rate (e.g. Figure 4 in Ruskin et al. 1998). Of the eleven GP neurons recorded during cocaine treatment, one fell into this latter category. Notably, although cocaine caused a $66 \%$ decrease in overall firing rate in this one case, the unit still displayed the cocaine-induced reduction in oscillatory period similar to the typical "firing rate-increasing" population (Figure 6). Similar period reductions have been noted in rare type II units demonstrating firing rate decreases after D-amphetamine or direct-acting $D_{1} / D_{2}$ agonists (unpublished results). 
Table 1. Expression of Multisecond Periodicities in GP Neurons

\begin{tabular}{|c|c|c|c|c|c|}
\hline & Before/After & Before/After & Before/After & Before/After & \\
\hline Treatment & $+l+$ & $-1+$ & $+1-$ & $-1-$ & $\mathbf{n}$ \\
\hline GBR-12909 (3.2) & 78 & 11 & 11 & 0 & 9 \\
\hline Desipramine $(2.0,5.0)$ & 68 & 16 & 10 & 5 & 19 \\
\hline Fluoxetine (3.2) & 67 & 22 & 11 & 0 & 9 \\
\hline Cocaine (3.2) & 82 & 9 & 0 & 9 & 11 \\
\hline
\end{tabular}

Most GP units have significant multisecond periodicities both before and after monoamine uptake blockade. Each GP unit is classified into one of four categories based on the expression of slow oscillations in firing rate 'before' and 'after' drug injection. The 'after' category refers to the 5-10 min post-injection time range. ' + ' and ' - ' indicate the presence and absence, respectively, of statistically significant periodic activity in the $1.5-60 \mathrm{~s}$ range of periods. Numbers are percentages. For example, in the rats receiving fluoxetine, $67 \%$ of neurons had oscillations both in baseline and after drug injection, $22 \%$ did not have baseline oscillations but did have oscillations after drug, $11 \%$ had baseline oscillations but no oscillations after drug, and no neurons were without oscillations in baseline and drug epochs. Numbers in parentheses are i.v. doses in $\mathrm{mg} / \mathrm{kg}$.

Because cocaine caused reductions in oscillatory period similar to those caused by the selective DA uptake blocker GBR-12909, we attempted to confirm the DAergic mediation of cocaine effects with DA receptor antagonists. A pretreatment with combined $\mathrm{D}_{1}$ and $\mathrm{D}_{2}$ antagonists (for complete antagonism of both DA receptor subfamilies) completely prevented the effects of cocaine on oscillatory period (Figure 3, Panel E). The combined DA antagonist treatment did not change oscillatory period by itself (Figure 3, Panel E), but did significantly reduce oscillatory power compared to baseline. After subsequent cocaine injection, oscillatory power was no longer significantly different from baseline (Figure 4), although it was also not significantly different from that after DA antagonist alone ( $p=.11$, Tukey test).

Pretreatment with the non-competitive NMDA receptor antagonist MK-801 also prevented the effects of cocaine on oscillatory period, without having any effect by itself on period (Figure 3, Panel F). In addition, MK801 by itself significantly decreased oscillatory power, and power remained reduced after subsequent injection of cocaine (Figure 4 ).

In the 5-10 min post-drug time range, freely-moving rats injected with $3.2 \mathrm{mg} / \mathrm{kg}$ cocaine i.v. demonstrated strong sniffing (directed downward, upward, or at the walls of the chamber), concomitant with almost contin-

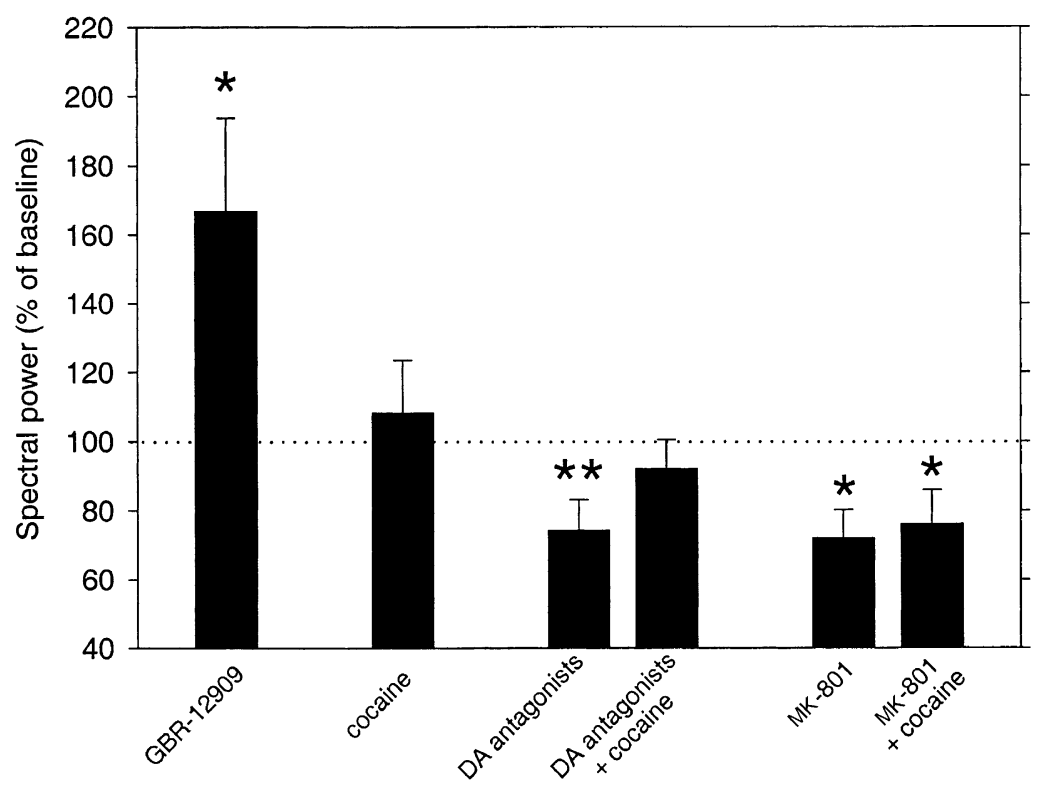

Figure 4. The effect of GBR-12909 or cocaine on oscillatory power, and the effect of pretreatment with DA receptor or NMDA receptor antagonists. Drug effects on spectral power (taken from the Lomb algorithm) are expressed as percent of baseline power. Significant main effects were found for GBR-12909 (F = 3.8, $p<.05$ ), combined injection of SCH-23390 and eticlopride $\left(\mathrm{D}_{1}\right.$ and $\mathrm{D}_{2}$ antagonists) followed by cocaine $(\mathrm{F}=6.0, p<.01)$ and MK-801 followed by cocaine $(\mathrm{F}=6.1, p<.01)$. Only data for the 5-10 min time range are shown. ${ }^{*} p<.05,{ }^{* *} p<.02$. GBR-12909 also demonstrated a trend $(p=.10)$ to increased power in the 15-20 min time range (not shown). ' $n$ ' for groups same as Figure 3. 

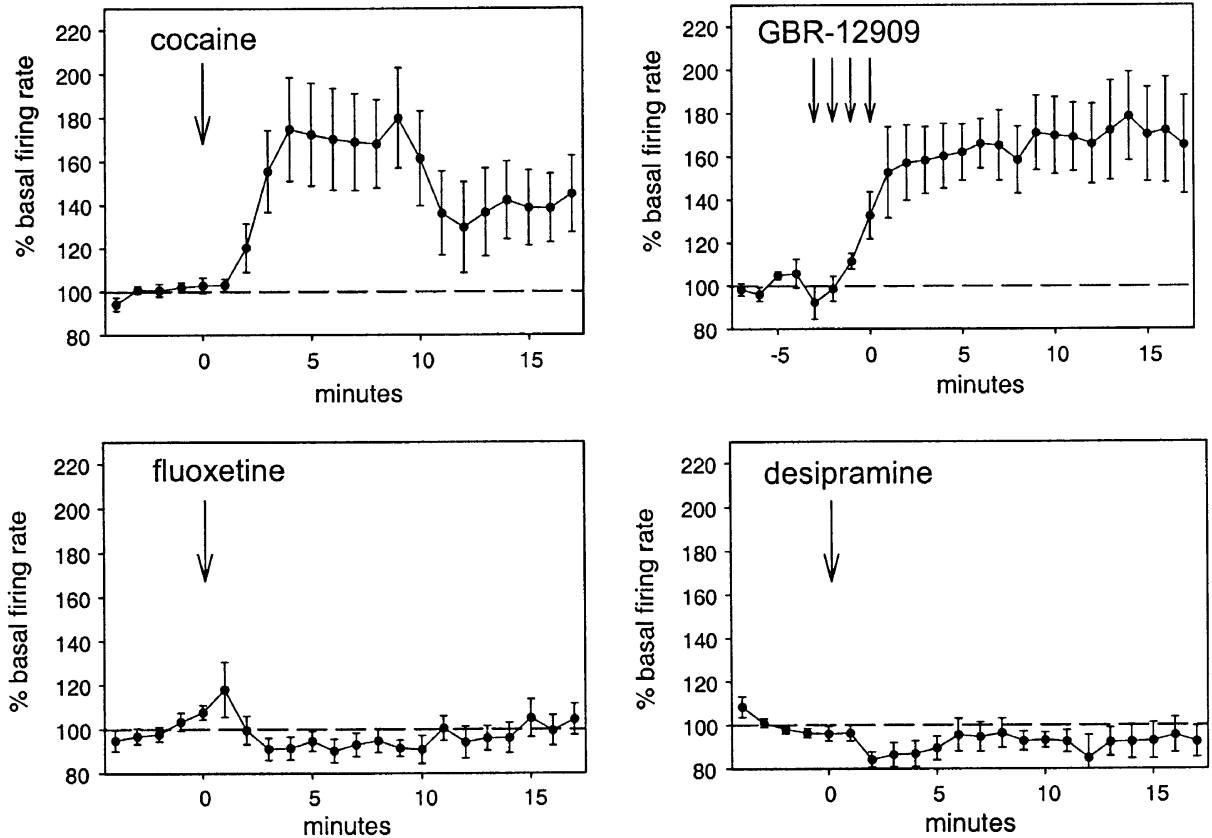

Figure 5. The effect of monoamine uptake blockers on mean firing rates in the GP. Firing rates are shown as minuteby-minute mean \pm SEM, and are expressed as percent of baseline firing rate. Cocaine $(3.2 \mathrm{mg} / \mathrm{kg}, n=11)$ and GBR-12909 (3.2 $\mathrm{mg} / \mathrm{kg}, n=9$ ) increase GP firing rates up to approximately $180 \%$ of baseline. Note the shorter-acting effect of cocaine, consistent with its shorter-acting effect on multisecond periodicities. Fluoxetine $(3.2 \mathrm{mg} / \mathrm{kg}, n=9)$ and desipramine $(5.0 \mathrm{mg} / \mathrm{kg}, n=$ 10) caused minor decreases in firing rate. Desipramine had similar effects at $2.0 \mathrm{mg} / \mathrm{kg}$ (not shown). In cases where GBR-12909 was injected as a single bolus dose $(n=3)$, the time of this injection is aligned with the first of the four arrows.

uous locomotion or rearing. Drug effects noticeably waned by the 15-20 min post-injection time range: rats occasionally sniffed, locomoted, or groomed, but were still most of the time.

\section{DISCUSSION}

Previous studies from our laboratory demonstrated that DA strongly modulates multisecond periodicities that are present in the spike trains of many GP neurons in awake rats. To extend these findings, the effects of other monoamines on GP firing rate oscillations were investigated. The present data show that augmenting NEergic or 5-HTergic transmission alone has minor effects on multisecond periodicities, and confirm the dominance of the DA system. These data also confirm that DA has a primary role among the monoamines for inducing increases in average firing rate in the GP (Bergstrom and Walters 1981). Because the DA agonists used in this study are indirect-acting, i.e. they augment levels of endogenous transmitter rather than directly activate receptors, these data demonstrate that an increase in endogenous DA is as effective in modulating the period and regularity (power) of slow oscillations in the GP as is direct activation of DA receptors by exogenous agonists. Work with D-amphetamine and meth- ylphenidate supports this conclusion (Baek et al. 1999; Ruskin et al. 2001). These results are therefore relevant to drug abuse and addictive processes in humans, since abused catecholaminergic stimulants (including cocaine) act via endogenous transmitter.

The effects of GBR-12909 or cocaine treatment on GP firing rate and pattern, prevented and reversed by DA receptor antagonists, are consistent with the marked increases in DA overflow caused by these drugs at or around the presently tested doses (Church et al. 1987; Baumann et al. 1994; Fink-Jensen et al. 1994; Engberg et al. 1997). However, our observations indicate that other transmitter systems are involved in these electrophysiological effects. For instance, pretreatment with the NMDA receptor antagonist MK-801 blocked cocaine's reduction of oscillatory period, suggesting that ongoing activity at NMDA receptors is necessary for this DAinduced effect. This type of NMDA receptor dependence has been noted for other electrophysiological effects of DA agonists in the basal ganglia (Kelland and Walters 1992; Allers et al. 1996; Huang et al. 1998), as well as for other measures of basal ganglia activity (reviewed in Huang et al. 1998). In addition, a comparison of the effects of GBR-12909 and cocaine reveals that although both drugs reduced oscillatory period (from $\sim 30 \mathrm{~s}$ to $\sim 10 \mathrm{~s}$ ), only GBR-12909 increased oscillation power. Direct-acting DA receptor agonists such as apo- 

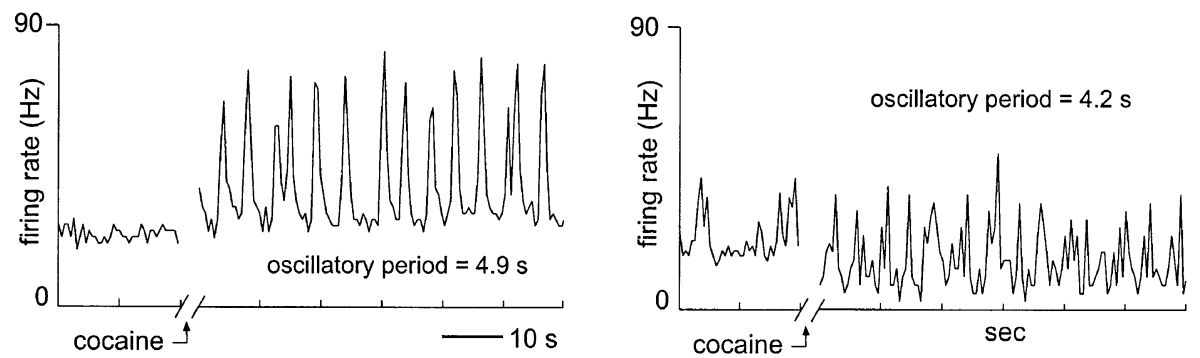

Figure 6. The lack of relationship between stimulant effects on oscillatory period effects on net firing rate. Tick marks on the time axes indicate 10-s increments. One minute of binned spiking activity (from several minutes after i.v. cocaine injection) is shown, along with a short epoch of baseline activity for comparison of overall firing rate. Both units have typical, short-period oscillations after cocaine, even though the effects of cocaine on overall firing rate in the two neurons are opposite. The net firing rate of the unit on the left increased by $57 \%$ compared to baseline, while the unit on the right responded to cocaine with a $66 \%$ decrease in net firing rate.

morphine also increase power (Ruskin et al. 1999a). Because cocaine blocks uptake of NE and 5-HT, in addition to DA uptake, these results suggest that increased NE or 5-HT levels might diminish DA-mediated increases in oscillatory power (while not affecting DA modulation of oscillatory period). A number of studies have demonstrated a reduction in DA-stimulated behaviors by 5-HTergic or NEergic systems (Ungerstedt 1971; Mabry and Campbell 1973; Grabowska 1976; Fox and Brotchie 1996, 2000; Henry et al. 1998; Rocha et al. 1998; Gainetdinov et al. 1999). More generally, the present results demonstrate that the period and power of multisecond oscillations in the basal ganglia can be independently affected, in agreement with a previous study of DA agonist effects in another basal ganglia region, the entopeduncular nucleus (Ruskin et al. 1999b).

In addition to being modulated by increased DAergic transmission, ongoing multisecond oscillations appear to partially depend on tonic DAergic and glutamatergic activity. This dependence is reflected in the significant $\sim 15 \%$ decrease in oscillatory power after treatment with combined $D_{1} / D_{2}$ receptor antagonists or a NMDA receptor antagonist. Interestingly, although these treatments blocked the effects of cocaine on oscillatory period, they did not affect oscillatory period when given alone (similarly, oscillatory period in the subthalamic nucleus is not affected by decreased DAergic transmission due to lesion of midbrain DA neurons (Allers et al. 2000)). Since spectral power reflects oscillatory regularity, the results suggest that tonic DAergic and glutamatergic (NMDA) tone partially supports the regularity of ongoing slow oscillations. The level of DAergic tone in these immobilized rats is not likely to be higher than normal, since firing rates of substantia nigra pars compacta DA neurons are not significantly increased in immobilized rats compared to anesthetized rats (Melis et al. 1998), and since, regarding multisecond oscillations, the effects of DA antagonists alone (no change in period, small-magnitude power decrease) are minor compared with the effects of DA agonists. On the other hand, DA antagonists have striking effects when administered during pharmacologically-stimulated DA release (Figure 1). Other transmitter systems are also involved in supporting slow oscillatory power: general anesthetics (specifically, urethane and ketamine/xylazine), which affect a number of transmitter systems, reduce spectral power in GP spike trains in the $1.5-60$ s range by 50 to $60 \%$ (unpublished results), leading to a severe decrease in the incidence of statistically significant multisecond oscillations (Ruskin et al. 1999a; Ruskin et al. 2001).

The decrease in spectral power after DA antagonist administration was partially reversed by subsequent cocaine injection. Such data suggest that the nondopaminergic effects of cocaine can increase multisecond spectral power in the absence of DAergic tone. Together with the comparison of the effects of GBR-12909 and cocaine (which indicate a decrease of DA-stimulated multisecond spectral power), the results suggest a 'normalizing' effect of 5-HT or NE release on the DAergic modulation of slow oscillatory power. This effect of 5-HT or NE appears to depend on tonic NMDA receptor activity, since there is no increase in spectral power after cocaine injection after prior injection of MK-801.

Multisecond periodicities have been noted in blood pressure (termed Mayer waves) and heart rate (Barnes and Burnham 1969; Mautner-Huppert et al. 1989). It is possible that these peripheral oscillations might somehow drive central multisecond oscillations. However, it has recently been reported that desipramine, administered i.v. to awake rats at one of the presently tested doses $(2.0 \mathrm{mg} / \mathrm{kg})$, induces particularly strong arterial pressure oscillations in the 10-s range (Bertram et al. 1999). Since, in the present study, desipramine produced (at best) inconsistent effects on oscillatory period and power, it seems probable that the multisecond oscillations in activity in the basal ganglia are not simply secondary to peripheral cardiovascular oscillations. In 
addition, the DA receptors that modulate basal ganglia multisecond oscillations are located centrally, not peripherally (Ruskin et al. 1999a; Ruskin et al. 1999c).

In terms of mechanisms of the DAergic modulation of slow oscillations, it is important to note that drug effects on oscillatory period were not related to drug effects on overall firing rate. In other neuronal systems and frequency ranges, the rate of oscillatory spiking activity or the rate of periodic bursting is positively correlated with the level of depolarization (Leresche et al. 1991; Beurrier et al. 1999; Koshiya and Smith 1999), implying a voltage-dependent mechanism. Such a relationship is not apparent for multisecond periodicities in the GP. Although treatments that increased oscillatory frequency also increased mean firing rate in a majority of neurons, some neurons demonstrated an increased oscillatory frequency simultaneously with a decreased mean firing rate. Additionally, in an earlier study of multisecond oscillations in the GP (Ruskin et al. 1999c), $\mathrm{D}_{1}$ agonists were found to increase oscillatory frequency without having consistent effects on mean firing rate, while $\mathrm{D}_{2}$ agonists modestly increased firing rate without having consistent effects on oscillations. Therefore, oscillatory period does not appear to be voltage-related for GP neurons when considered as a single population. Similar conclusions have been made about neurons in the entopeduncular nucleus and substantia nigra pars reticulata (Ruskin et al. 1999a). Oscillatory frequency might be positively linked to depolarization in the predominant subpopulation of GP neurons that increase average firing rate after combined $D_{1} / D_{2}$ receptor activation, and these neurons might drive (via local axon collaterals) other GP neurons to oscillate at a similar speed. However, this scenario seems unlikely, since there is no correlation between oscillation frequency and net firing rate (either absolute or percent of baseline) after cocaine or GBR-12909 treatment in the subpopulation with increases in average firing rate (data not shown). If oscillatory period in the GP is not related to membrane voltage, then the mechanisms by which DA modulates net firing rate and oscillatory period in the GP must differ.

Regarding the mechanism of generation of slow oscillations, if these oscillations (regardless of period) can occur in the GP within a wide range of membrane voltages, then they are likely not generated by voltagedependent mechanisms within the GP. Instead, they might be generated in afferent structures and transmitted synaptically to the GP, or they might arise from network mechanisms within the GP or between the GP and other structures. There is some evidence for this last hypothesis, as reverberating oscillations have been found in organotypic co-cultures of GP and subthalamic nucleus, albeit at shorter periods (Plenz and Kitai 1999). Also, network mechanisms are clearly involved in correlating (if not also generating) multisecond oscil- latory activity, as spatially distant basal ganglia neurons can have similar periods with constant phase relationships (Allers et al. 1999; Ruskin et al. 2000).

Although wavelet analysis has been increasingly utilized for analysis of electroencephalographic signals, fewer studies have used this technique for analysis of single unit data. Many of these latter studies have utilized wavelets for spike sorting (Zouridakis and Tam 1997) or denoising (Koshiya and Smith 1999) applications, rather than for analyzing neuronal spiking in the time-frequency domain (Przybyszewski 1991; Lewalle et al. 1995). Here, wavelet analysis successfully revealed multisecond periodicities in GP spiking activity, and complemented our standard spectral analysis technique, the Lomb periodogram. Apart from the obvious differences in the methodologies (producing time information in the wavelet scalogram, and statistical information in the Lomb periodogram), these two techniques routinely indicated similar oscillatory activity for a given data segment. For instance, in Figure 2, Panel A, center, both techniques indicate a strong oscillation of $\sim 12 \mathrm{~s}$ after cocaine injection, while in the baseline epoch depicted in Figure 2, Panel B, left, both methods indicate a lack of substantial multisecond oscillatory activity. Yet there are subtle differences between the two methods, such as the more sensitive detection of the 25-s periodicity by the Lomb periodogram in Figure 2, Panel A, left. Possibly the most enlightening use of the wavelet scalogram for the present data is to reveal the nature of the multiple, statistically significant spectral peaks which are present in the Lomb periodogram in some cases. In Figure 2, Panel B, middle, the Lomb spectrum of a post-cocaine epoch shows a number of spectral peaks with periods ranging from 3.7 to $6.6 \mathrm{~s}$ centered on a powerful main peak of $4.9 \mathrm{~s}$. Inspection of the corresponding scalogram reveals that the period of the neuron's strong oscillatory activity was continuously shifting within the analyzed time, with an overall decrease in period. Hence, in this case the multiple peaks in the Lomb spectrum arise from the period variation of the predominant oscillation over time. Alternatively, concerning the weaker oscillatory activity present in Figure 2, Panel B, right, the Lomb periodogram indicates three spectral peaks of nearly equal strength, at $7.7,14$, and $33 \mathrm{~s}$. The scalogram demonstrates that the $33 \mathrm{~s}$ oscillation was present throughout the analyzed segment, simultaneously with the 7.7 and 14 s oscillations, which themselves were segregated to early and later parts of the segment. Therefore, the presence of multiple spectral peaks can indicate simultaneous oscillations and/or shifts in oscillation period over time.

Periodic oscillations in activity in the seconds-tominutes range have been noted in many biological systems, and have been attributed with various functions. In particular, oscillatory fluctuations in intracellular levels of the second-messenger calcium are more effi- 
cient than simple changes in mean calcium level for inducing gene expression, enzymatic activity and hormone secretion in some in vitro systems (Holl et al. 1988; Matozaki et al. 1990; Dolmetsch et al. 1998; De Koninck and Schulman 1998; Li et al. 1998; Charles et al. 1999; Narenjkar et al. 1999; Tomić et al. 1999). Furthermore, it was hypothesized in some of these studies that the calcium-mediated influence on effector systems is related to oscillatory frequency. The oscillations in neuronal firing rate in the present study presumably drive slow variations in intracellular calcium concentration which might be functionally significant; however, they are likely to have effects beyond signal transduction, namely at systems and behavioral levels. Such prominent temporal patterning in basal ganglia spiking activity is likely to be associated with corresponding patterning in activity of other forebrain regions (via the basal ganglia ouput pathways), and so to modulation of cognition and behavior. Cocaine and GBR-12909 (as well as D-amphetamine and methylphenidate) induce stereotyped sniffing and oral movements, stereotyped locomotion, and perseveration during the execution of learned motor behaviors (Kokkinidis and Anisman 1976; Ridley et al. 1981; Mueller et al. 1989; Paulus et al. 1993; Rosenzweig-Lipson et al. 1994; present results) in rodents and non-human primates. Cocaine or amphetamine intoxication in humans can lead to similar behaviors (reviewed in Lyon and Robbins 1975). GP neurons are well-characterized as having alterations in firing rate in relation to movement, so that multisecond oscillations in pallidal firing rate might relate to some specific periodicity of limb or body movement, or motor state. Such relationships will need to be characterized in studies of freely-moving animals. In addition to motor effects, cocaine, amphetamine and methylphenidate all produce a 'high' or 'rush' at sufficient doses in humans, and continued usage typically leads to addiction. In studies of rodents and non-human primates, all these stimulants as well as GBR-12909 (Bergman et al. 1989; Roberts 1993) support self-administration. The similarity of the effects of these drugs on basal ganglia multisecond oscillations, and their common effects on these behaviors, suggests that abnormal multisecond patterning in the forebrain (with shifted frequency range and/or increased power) might relate to the acute motor and affective/cognitive effects of stimulant treatment, and might represent a state of brain activity which induces or augments addictive processes.

\section{REFERENCES}

Allers KA, Kreiss DS, Twery MJ, Juncos JL, Walters JR (1996): The role of glutamate in D1 agonist induced increases of subthalamic nucleus neuronal firing rate: normal vs. 6-OHDA lesioned rats. Soc Neurosci Abstr 22:1200
Allers KA, Kreiss DS, Walters JR (2000): Multisecond oscillations in the subthalamic nucleus: effects of apomorphine and dopamine cell lesion. Synapse 38:38-50

Allers KA, Ruskin DN, Bergstrom DA, Molnar LR, Walters JR (1999): Correlations of multisecond oscillations in firing rate in pairs of basal ganglia neurons. Soc Neurosci Abstr 25:1929

Baek D, Ruskin DN, Bergstrom DA, Walters JR (1999): D-amphetamine (AMPH) and cocaine increase the speed of multisecond oscillations in globus pallidus (GP) unit activity. Soc Neurosci Abstr 25:1819

Barbieri EJ, Ferko AP, DeGregorio GJ, Ruch EK (1992): The presence of cocaine and benzoylecgonine in rat cerebrospinal fluid after the intravenous administration of cocaine. Life Sci 51:1739-1746

Barnes CD, Burnham E (1969): The reflection of third order blood pressure waves in the lumbar monosynaptic reflex. Brain Res 13:183-186

Baumann MH, Char GU, De Costa BR, Rice KC, Rothman RB (1994): GBR12909 attenuates cocaine-induced activation of mesolimbic dopamine neurons in the rat. J Pharmacol Exp Ther 271:1216-1222

Bergman J, Madras BK, Johnson SE, Spealman RD (1989): Effects of cocaine and related drugs in nonhuman primates. III. Self-administration by squirrel monkeys. J Pharmacol Exp Ther 251:150-155

Bergstrom DA, Walters JR (1981): Neuronal responses of the globus pallidus to systemic administration of $d$-amphetamine: investigation of the involvement of dopamine, norepinephrine, and serotonin. J Neurosci 1:292-299

Bertram D, Barrès C, Julien C (1999): Effect of desipramine on spontaneous arterial pressure oscillations in the rat. Eur J Pharmacol 378:265-271

Beurrier C, Congar P, Bioulac B, Hammond C (1999): Subthalamic nucleus neurons switch from single-spike activity to burst-firing mode. J Neurosci 19:599-609

Charles AC, Piros ET, Evans CJ, Hales TG (1999): L-type $\mathrm{Ca}^{2+}$ channels and $\mathrm{K}^{+}$channels specifically modulate the frequency and amplitude of spontaneous $\mathrm{Ca}^{2+}$ oscillations and have distinct roles in prolactin release in $\mathrm{GH}_{3}$ cells. J Biol Chem 274:7508-7515

Church WH, Justice Jr JB, Byrd LD (1987): Extracellular dopamine in rat striatum following uptake inhibition by cocaine, nomifensine and benztropine. Eur J Pharmacol 139:345-348

De Koninck P, Schulman H (1998): Sensitivity of CaM kinase II to the frequency of $\mathrm{Ca}^{2+}$ oscillations. Science 279:227-230

Dolmetsch RE, Xu K, Lewis RS (1998): Calcium oscillations increase the efficiency and specificity of gene expression. Nature 392:933-936

Engberg G, Elverfors A, Jonason J, Nissbrandt H (1997): Inhibition of dopamine re-uptake: significance for nigral dopamine neuron activity. Synapse 25:215-226

Fink-Jensen A, Ingwersen SH, Nielsen PG, Hansen L, Nielsen EB, Hansen AJ (1994): Halothane anesthesia enhances the effect of dopamine uptake inhibition on interstitial levels of striatal dopamine. Naunyn Schmeidebergs Arch Pharmacol 350:239-244

Fox SH, Brotchie JM (1996): Normethylclozapine potentiates 
the action of quinpirole in the 6-hydroxydopamine lesioned rat. Eur J Pharmacol 301:27-30

Fox SH, Brotchie JM (2000): $5-\mathrm{HT}_{2 \mathrm{C}}$ receptor antagonists enhance the behavioural response to dopamine $D_{1}$ receptor agonists in the 6-hydroxydopamine-lesioned rat. Eur J Pharmacol 398:59-64

Gainetdinov RR, Wetsel WC, Jones SR, Levin ED, Jaber M, Caron MG (1999): Role of serotonin in the paradoxical calming effects of psychostimulants on hyperactivity. Science 283:397-401

Grabowska M (1976): The involvement of serotonin in the mechanism of central action of apomorphine. Pol J Pharmacol Pharm 28:389-394

Henry B, Crossman AR, Brotchie JM (1998): Characterization of enhanced behavioral responses to L-DOPA following repeated administration in the 6-hydroxydopaminelesioned rat model of Parkinson's disease. Exp Neurol 151:334-342

Holl RW, Thorner MO, Mandell GL, Sullivan JA, Sinha YM, Leong DA (1988): Spontaneous oscillations of intracellular calcium and growth hormone secretion. J Biol Chem 263:9682-9685

Huang K-X, Bergstrom DA, Ruskin DN, Walters JR (1998): N-methyl-D-aspartate receptor blockade attenuates D1 dopamine receptor modulation of neuronal activity in rat substantia nigra. Synapse 30:18-29

Kaneoke Y, Vitek JL (1996): Burst and oscillation as disparate neuronal properties. J Neurosci Meth 68:211-223

Kelland MD, Soltis RP, Anderson LA, Bergstrom DA, Walters JR (1995): In vivo characterization of two cell types in the rat globus pallidus which have opposite responses to dopamine receptor stimulation: comparison of electrophysiological properties and responses to apomorphine, dizocilpine, and ketamine anesthesia. Synapse 20:338-350

Kelland Sr MD, Walters JR (1992): Apomorphine-induced changes in striatal and pallidal neuronal activity are modified by NMDA and muscarinic receptor blockade. Life Sci 50:PL179-184

Kokkinidis L, Anisman H (1976): Interaction between cholinergic and catecholaminergic agents in a spontaneous alternation task. Psychopharmacology 48:261-270

Koshiya N, Smith JC (1999): Neuronal pacemaker for breathing visualized in vitro. Nature 400:360-363

Leresche N, Lightowler S, Soltesz I, Jassik-Gerschenfeld D, Crunelli V (1991): Low-frequency oscillatory activities intrinsic to rat and cat thalamocortical cells. J Physiol 441:155-174

Lewalle J, Peek FW, Murphy SJ (1995): Wavelet analysis of olfactory nerve response to stimulus. J Theor Biol 177:215-236

Li W-h, Llopis J, Whitney M, Zlokarnik G, Tsien RY (1998): Cell-permeant caged InsP3 ester shows that $\mathrm{Ca}^{2+}$ spike frequency can optimize gene expression. Nature 392:936-941

Lyon M, Robbins T (1975): The action of central nervous system stimulant drugs: a general theory concerning amphetamine effects. In Essman WB, Valzelli L (eds), Current Developments in Psychopharmacology, Vol. 2. New York, Spectrum Publications, Inc., pp 80-163

Mabry PD, Campbell BA (1973): Serotonergic inhibition of catecholamine-induced behavioral arousal. Brain Res 49:381-391

Matozaki T, Göke B, Tsunoda Y, Rodriguez M, Martinez J, Williams JA (1990): Two functionally distinct cholecystokinin receptors show different modes of actions on $\mathrm{Ca}^{2+}$ mobilization and phospholipid hydrolysis in isolated rat pancreatic acini. J Biol Chem 265:6247-6254

Mautner-Huppert D, Haberl RL, Dirnagl U, Villringer A, Schmiedek P, Einhäupl K (1989): B-waves in healthy persons. Neurol Res 11:194-196

Melis M, Mereu G, Lilliu V, Quartu M, Diana M, Gessa GL (1998): Haloperidol does not produce dopamine cell depolarization-block in paralyzed, unanesthetized rats. Brain Res 783:127-132

Mueller K, Hollingsworth EM, Cross DR (1989): Another look at amphetamine-induced stereotyped locomotor activity in rats using a new statistic to measure locomotor stereotypy. Psychopharmacology 97:74-79

Narenjkar J, Marsh SJ, Assem ESK (1999): The characterization and quantification of antigen-induced $\mathrm{Ca}^{2+}$ oscillations in a rat basophilic leukaemia cell line (RBL-2H3). Cell Calcium 26:261-269

Paulus MP, Callaway CW, Geyer MA (1993): Quantitative assessment of the microstructure of rat behavior: II. Distinctive effects of dopamine releasers and uptake inhibitors. Psychopharmacology 113:187-198

Plenz D, Kitai ST (1999): A basal ganglia pacemaker formed by the subthalamic nucleus and external globus pallidus. Nature 400:677-682

Przybyszewski AW (1991): An analysis of the oscillatory patterns in the central nervous system with the wavelet method. J Neurosci Meth 38:247-257

Ridley RM, Baker HF, Haystead TA (1981): Perseverative behaviour after amphetamine; dissociation of response tendency from reward association. Psychopharmacology 75:283-286

Roberts DCS (1993): Self-administration of GBR-12909 on a fixed-ratio and progressive ratio schedule in rats. Psychopharmacology 111:202-206

Rocha BA, Scearce-Levie K, Lucas JJ, Hiroi N, Castanon N, Crabbe JC, Nestler EJ, Hen R (1998): Increased vulnerability to cocaine in mice lacking the serotonin-1B receptor. Nature 393:175-178

Rosenzweig-Lipson S, Hesterberg P, Bergman J (1994): Observational studies of dopamine D1 and D2 agonists in squirrel monkeys. Psychopharmacology 116:9-18

Ruskin DN, Bergstrom DA, Kaneoke Y, Patel BN, Twery MJ, Walters JR (1999a): Multisecond oscillations in firing rate in the basal ganglia: Robust modulation by dopamine receptor activation and anesthesia. J Neurophysiol 81:2046-2055

Ruskin DN, Bergstrom DA, Shenker A, Freeman LE, Baek D, Walters JR (2001): Drugs used in the treatment of attention-deficit/hyperactivity disorder affect postsynaptic firing rate and oscillation without preferential dopamine autoreceptor action. Biol Psychiatry, 49:340-350

Ruskin DN, Bergstrom DA, Walters JR (1999b): Firing rates and multisecond oscillations in the rodent entopeduncular nucleus (EPN): effects of dopamine (DA) agonists and nigrostriatal lesion. Soc Neurosci Abstr 25:1929

Ruskin DN, Bergstrom DA, Walters JR (1999c): Multisecond 
oscillations in firing rate in the globus pallidus: synergistic modulation by D1 and D2 dopamine receptors. J Pharmacol Exp Ther 290:1493-1501

Ruskin DN, Bergstrom DA, Walters JR (2000): Spatially distant neurons in the basal ganglia can demonstrate correlated multisecond periodicities in firing rate in vivo. Winter Conference on Brain Research; Breckenridge, Colorado.

Ruskin DN, Rawji SS, Walters JR (1998): Effects of full $D_{1}$ dopamine receptor agonists on firing rates in the globus pallidus and substantia nigra pars compacta in vivo: tests for $\mathrm{D}_{1}$ receptor selectivity and comparisons in the partial agonist SKF 38393. J Pharmacol Exp Ther 286:272-281
Tomić M, Koshimizu T-a, Yuan D, Andric SA, Zivadinovic D, Stojilkovic SS (1999): Characterization of a plasma membrane calcium oscillator in rat pituitary somatotrophs. J Biol Chem 274:35693-35702

Ungerstedt U (1971): Striatal dopamine release after amphetamine or nerve degeneration revealed by rotational behavior. Acta Physiol Scand Suppl 367:49-68

Wichmann T, Bergman H, Kliem MA, Soares J, DeLong MR (1999): Low-frequency oscillatory discharge in the primate substantia nigra pars reticulata $(\mathrm{SNr})$ in the normal and parkinsonian state. Soc Neurosci Abstr 25: 1928

Zouridakis G, Tam DC (1997): Multi-unit spike discrimination using wavelet transforms. Comput Biol Med 27:9-18 